\title{
Review finds failure rates lower for delayed loaded submerged dental implants
}

\author{
Abstracted from \\ Chrcanovic BR, Albrektsson T, Wennerberg A. \\ Immediately loaded non-submerged versus delayed loaded submerged dental implants: a meta-analysis. \\ Int J Oral Maxillofac Surg 2015; 44: 493-506. \\ Address for correspondence: Dr BR Chrcanovic, Department of Prosthodontics, Faculty of Odontology, \\ Malmö University, Malmö, Sweden. E-mail: bruno.chrcanovic@mah.se
}

\section{Question: How do the survival rate and post-operative complications of non-submerged immediately loaded dental implants compare with those of submerged delayed loaded implants?}

Data sources PubMed, Web of Science, Cochrane Oral Health Group Trials Register, Clinical Trials.gov, Center Watch.com, Clinical Connection.com

Study selection Randomised and non-randomised studies were included comparing implant failure rates in any group of patients receiving submerged versus immediately loaded non-submerged dental implants. Selection was conducted independently by three reviewers.

Data extraction and synthesis Titles and abstracts of all reports identified through the electronic searches were read independently by the three authors. Studies were selected based on inclusion and exclusion criteria, with disagreements resolved through discussion. Study quality was assessed using the Cochrane risk of bias tool. Implant failure and post-operative infection were the dichotomous outcome measures evaluated. Weighted mean differences (WMD) were calculated and meta-analysis conducted.

Results Twenty eight studies, consisting of six randomized clinical trials, 14 controlled clinical trials and eight retrospective analyses were included. 23 studies were considered to be at high risk of bias, one at moderate risk and four at low risk of bias. The relative risk (RR) of failure was higher in immediately loaded implants RR $=1.78(95 \%$ $\mathrm{Cl} ; 1.12-2.83$ ). The number needed to treat (NNT) to prevent one patient having an implant failure is 50 (95\% Cl; $25-100)$. Analysis suggests the possibility of publication bias.

Conclusions The difference between immediately loading and delayed loading of an implant statistically affected the implant failure rate. No statistically significant effects on the occurrence of postoperative infection were observed between the two techniques. Results should be interpreted with caution due to lack of control of confounding factors, the retrospective design of some studies included and the small cohort sizes within the studies.

\section{Commentary}

Since the revolutionary discovery of osseointegration, dental implants have been historically placed according to the Brånemark protocol. $^{1}$ This method involved delicate surgical technique, whereby the implant was allowed to heal in situ without the burden of loading for a period of at least three to four months. This practice was aimed towards reducing the incidence of infection, ${ }^{2}$ reducing micro-movement and increasing primary stability of the implant; ${ }^{3}$ factors which would promote osseointegration and reduce the risk of fibrous encapsulation of the implant. As times have moved on, so have the wishes and expectations of our patients. Nowadays, patient-centred outcomes such as reduced treatment times, surgical interventions and costs are factors seen as desirable. ${ }^{4}$ Indeed, for patients suffering tooth loss and for those who are inexperienced with removable prostheses, it can be hard to tolerate a submerged healing period. As such, the advantages of an immediate loading protocol have fuelled much research in this area in recent years.

The meta-analysis by Chrcanovic et al. ${ }^{5}$ is well written and satisfies many of the criteria set out in the PRISMA appraisal tool. ${ }^{6}$ A review protocol is an important step to provide readers with a step-by-step methodology to enable transparency and reproducibility. Such a protocol enables replication of the analysis, which in turn imparts reliability of the analysis findings. However, the authors advise that a review protocol does not exist.

The authors utilise a detailed and repeatable search strategy and made efforts to make contact with authors in order to obtain missing data. Although Chrcanovic et al. did manually search a number of other journals, the authors note that only dental journals were included; and it is unclear why medical journals would be specifically excluded relating to dental implant placement. Ideally a search date should be given for manual journal searches in order to facilitate ease of replication of the analysis findings. The authors included a flow diagram detailing the numbers of studies screened, assessed for eligibility and included in the review, with reasons for exclusions given at each stage, however it may have been useful to have a reference list of all excluded studies. Equally it may have been useful to present confidence intervals for individual studies rather than the standard deviations (SD) that were given.

A quality assessment of the risk of bias across studies was performed using the Cochrane risk of bias tool; a detailed appraisal of bias risk across studies is also given. It would have been useful for added illustration to present this as a combined risk assessment figure, such as would be expected in a Cochrane review.

The systematic review highlights the difference in failure rate 
between implant placement techniques, with a good description of study limitations. The introduction provides a descriptive rationale of the advantages and disadvantages of single-stage implant surgery versus two-stage implant surgery and an explicit statement of the research question being addressed is given using the PICOS format. The problem with the placement of implants is that there are so many factors that influence success; from the detrimental relationship between smoking and peri-implant disease ${ }^{7}$ to the relationship between operator experience and implant success. ${ }^{8}$ Additionally, many studies in this field show considerable variability on what can be defined within the paradigm of 'immediate loading'. Clinicians can load implants within 48 hours $^{9}$ or delay the process for 20 days. ${ }^{10}$

A conventional meta-analysis is performed to combine the results of limited numbers of well-conducted RCTs with similar results, such that a summary effect can be synthesised. This is important because clinicians require concise, high quality guidance on whether or not an intervention can be effective. In order to increase sample sizes for the analysis, this meta-analysis included (non-randomised) controlled clinical trials and retrospective analysis, study designs which may be at high risk of bias. Many studies had small sample sizes and dissimilar results. Critics might argue that combining diverse studies in a meta-analysis may be an exercise in statistical alchemy, and may be akin to comparing apples with oranges. ${ }^{11}$

The very nature of many studies in dentistry preclude doubleblinding in the study design. To include a patient in a study, a clinician may assess primary stability ${ }^{12}$ before making a decision on which loading technique they may choose. It is this clinical assessment and decision that may result in subsequent selection bias.

Multiple confounding factors were present in the reviewed studies including small cohort sizes, cigarette smoking, splinting of implants, operator's surgical experience, immediate implant placement in extracted sockets versus delayed placement and short term follow-up periods. Overall Chrcanovic et al. give a good description of limitations and confounding factors which may have influenced the long-term outcomes analysed and recognise that these factors limit the potential to draw robust conclusions about the loading of implants.

\section{Practice points}

- It appears that delaying the loading of clinical implants increases their overall success rate.

\section{David McReynolds and Brett Duane Dublin Dental University Hospital, Dublin, Ireland}

1. Albrektsson $\mathrm{T}$, Brånemark $\mathrm{Pl}$, Hansson $\mathrm{HA}$, Lindström J. Osseointegrated titanium implants. Requirements for ensuring a long-lasting, direct bone-to-implant anchorage in man. Acta Orthop Scand 1981; 52: 155-170.

2. Heydenrijk K, Raghoebar GM, Meijer HJ, Van Der Reijden WA, Van Winkelhoff A] Stegenga B. Two-part implants inserted in a one-stage or a two-stage procedure. A prospective comparative study. / Clin Periodontol 2002; 29: 901-909

3. Roberts EW, Poon LC, Smith RK. Interface histology of rigid endosseous implants. J Oral Implantol 1986; 12: 406-416.

4. Gallucci GO, Benic Gl, Eckert SE, et al. Consensus statements and clinical recommendations for implant loading protocols. Int J Oral Maxillofac Implants 2014; 29: $287-290$.

5. Chrcanovic BR, Albrektsson T, Wennerberg A. Immediately loaded non-submerged versus delayed loaded submerged dental implants: a meta-analysis. Int / Oral Maxillofac Surg 2015; 44: 493-506. http://dx.doi.org/10.1016/j.ijom.2014.11.011

6. Moher D, Liberati A, Tetzlaff J, Altman DG; PRISMA Group. Preferred reporting items for systematic reviews and meta-analyses: the PRISMA statement. PLoS Med 2009; 6: e1000097. doi:10.1371/journal.pmed1000097

7. Aglietta M, Siciliano VI, Rasperini G, Cafiero C, Lang NP, Salvi GE. A 10-year restrospective analysis of marginal bone-level changes around implants in periodontally healthy and periodontally compromised tobacco smokers. Clin Oral Implants Res 2011; 22: 47-53.

8. Chrcanovic BR, Albrektsson T, Wennerberg A. Reasons for failures of oral implants. J Oral Rehabil 2014; 41: 443-476.

9. Testori T, Meltzer A, Del Fabbro M, et al. Immediate occlusal loading of Osseotite implants in the lower edentulous jaw. A multicenter prospective study. Clin Oral Implants Res 2004; 15: 278-284.

10. Ericsson I, Randow K, Nilner K, Peterson A. Early functional loading of Brånemark dental implants: 5-year clinical follow-up study. Clin Implant Dent Relat Res 2000; 2: 70-77.

11. Moayyedi P. Meta-analysis: Can we mix apples and oranges? Am J Gastroenterol 2004; 99: $2297-2301$.

12. Esposito M, Grusovin MG, Chew YS, Coulthard P, Worthington HV. Interventions for replacing missing teeth: 1- versus 2-stage implant placement. Cochrane Database Syst Rev 2009; 3: Art. No. CD006698. DOI: 10.1002/14651858.CD006698.pub2.

Evidence-Based Dentistry (2015) 16, 112-113. doi:10.1038/sj.ebd.6401134 